\title{
'Hunt the thimble': a study of the radiology of ingested foreign bodies
}

\author{
A. E. BOOTHROYD ${ }^{1}$ H. M. L. CARTY ${ }^{2}$ AND W. J. ROBSON ${ }^{3}$ \\ Departments of ${ }^{1}$ Paediatrics, ${ }^{2}$ Radiology and ${ }^{3}$ Accident and Emergency, Alder Hey \\ Children's Hospital, Liverpool, England
}

\section{SUMMARY}

The role of radiology in the management of patients with a history of ingested foreign bodies is reviewed in a retrospective study over a 2-year period in a Children's Hospital. If radiography was confined to those children who have a clinical history suggestive of foreign body impaction in the oesophagus, a foreign body aspiration or having swallowed a button battery, the work load and unnecessary radiation arising from this practice could be halved. This study demonstrates another misuse of radiological services.

\section{INTRODUCTION}

Children attending accident and emergency (A \& E) departments with a history of ingesting a foreign body are frequently referred for X-rays in order to locate the object. The aims of this retrospective study were to assess the incidence of these requests, the role of the investigation and the subsequent management of the patient.

\section{METHOD}

The records of patients who had attended with a history of foreign body ingestion during the years 1982 to 1984 were analysed. Details of their presenting systems, the nature of the object thought to have been ingested, the numbers and type of $\mathrm{X}$-rays

Correspondence: $\operatorname{Dr}$ H.M. L. Carty, Consultant Radiologist, Department of Radiology, Alder Hey Children's Hospital, Eaton Road, Liverpool L12 2AP, England 
requested, the timing of the $\mathrm{X}$-rays relative to the time of ingestion and the subseque clinical management of the patient were recorded.

\section{RESULTS}

During the 2-year period, 453 patients were identified as having a history of an ingested foreign body. Three hundred and ninety children presented primarily to the $A \& \frac{8}{8}$ department. Sixty-three children were admitted to hospital, of whom 35 were patients transferred from other hospitals in the region. The age range of the patients was from 18 months to 15 years with a peak incidence in pre-school children (Fig. 1). The se $\vec{x}$ incidence was equal. A total of $278(72 \%)$ children who presented to the A \& department were referred for X-ray. The stated indications for X-ray referral are show in Table 1. One hundred and fifty-five of those X-rayed were completely asymptomatic. The discovery of a foreign body on X-ray did not alter their management. In nis instance was a foreign body found in the chest.

The films initially requested were views of the neck, chest and abdomen. This necessitated one or two films depending on the size and cooperation of the chilctis Delayed films of the abdomen were taken in 35 children who had previously been X rayed at presentation. These films were taken at widely varying intervals which ranged from $24 \mathrm{~h}$ to 3 weeks after the time of the initial X-rays. The delayed films vrere requested by the casualty officers for the monitoring of the progress of the object of to

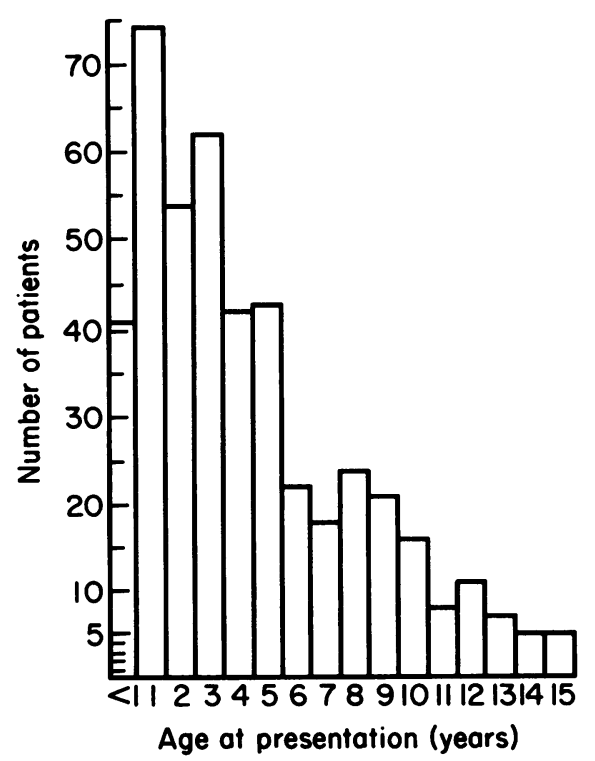

Fig. 1 Age distribution of children attending hospital with a history of an ingested foreign body. 
Table 1 Reasons for radiography in 278 patients attending the A \& E department

\begin{tabular}{lc}
\hline Clinical symptoms/signs & No. of patients \\
\hline Asymptomatic: no reason given* & 155 \\
Choking/coughing & 56 \\
Abdominal pain & 20 \\
Dysphagia/retrosternal pain & 20 \\
Vomiting & 11 \\
Details of symptoms not recorded & 12 \\
Bleeding from mouth & 4 \\
\hline
\end{tabular}

* Symptoms of cough, cynosis, etc., specifically denied. Information taken from records.

allay parental anxiety when the swallowed foreign body was not found in the stools. No impacted foreign body was demonstrated.

Table 2 lists the foreign bodies ingested. Ninety-four objects were clearly radiolucent, yet one-third of these children who ingested these objects were X-rayed and only 11 children had symptoms suggestive of aspiration. Symptoms which should suggest aspiration of a foreign body are: cyanosis, choking, gasping for breath, coughing and wheezing.

Table 2 Nature of foreign body in 453 patients

\begin{tabular}{lrll}
\hline Coin & 131 & Unknown & 4 \\
Plastic toy & 40 & Hairgrip & 3 \\
Nail/screw & 26 & Cigarette filter & 3 \\
Fishbone & 24 & Tooth & 3 \\
Marble & 23 & Ring pin & 2 \\
Ballbearing & 23 & Washer & 2 \\
Pin/safety pin & 23 & Metal wire & 2 \\
Glass & 18 & Pencil lead & 2 \\
Ring & 18 & Toy wheel & 1 \\
Food & 17 & Crayon & 1 \\
Sweet & 14 & Match & 1 \\
Pen top & 13 & Key & 1 \\
Button & 12 & Clothes peg spring & 1 \\
Earring & 10 & Cockroach & 1 \\
'Mastermind' peg & 6 & Meccano & 1 \\
Christmas decoration & 6 & Toy bell & 1 \\
Bone & 4 & Iron ('Monopoly') & 1 \\
Battery & 4 & Whistle & 1 \\
Paper clip & 4 & Cufflink & 1 \\
Pebble & 4 & Razor blade & 1 \\
\hline
\end{tabular}


Table 3 Outcome in 453 patients who ingested a foreign body

\begin{tabular}{lr}
\hline Home without review & 344 \\
Review in A \& E Department & 30 \\
Medical Outpatient Department & 1 \\
Surgical Outpatient Department & 4 \\
Admitted & 63 \\
Removal of foreign body in A \& E Department (then review) & 11 \\
\hline
\end{tabular}

The foreign bodies removed in the A \& E department were nine fish bones, one piece of curtain wire and a piece of glass. These were all in the orapharynx (Table 3 ).

Sixty-three patients were admitted to the wards. Twelve children were observed overnight -11 for abdominal pain and/or vomiting. One child was asymptomatic and was only kept in hospital as this child had been referred from another hospital in the region at quite a distance from Alder Hey. He was discharged the following morning $\overrightarrow{\vec{\omega}_{\omega}}$

The children admitted who subsequently required intervention for removal of the foreign body all had symptoms strongly suggestive of the site of obstruction (Table 4 )? Dysphagia, excessive salivation and/or retrosternal pain were associated with impaction in the oesophagus.

Table 4 Symptoms in patients requiring admission and intervention

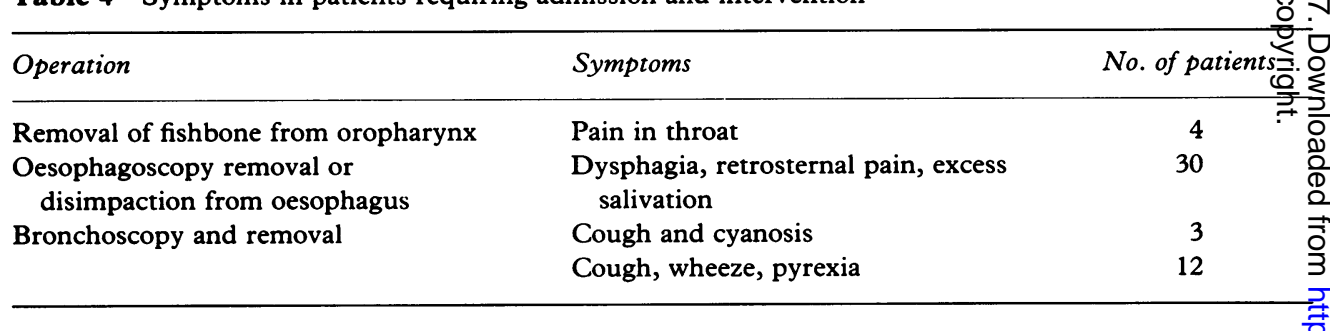

Fifteen children had impacted foreign bodies removed from the bronchus. Two cases presented acutely with a short history of coughing and cyanosis. The remaining $1 \frac{135}{5}$ children gave a history of cough, wheeze and pyrexia days or weeks after the ingestion of a foreign body.

Children who were clinically at risk of impaction of a foreign body having had repair of a tracheo-oesophageal fistula accounted for 10 admissions in the 2 years studied These admissions related to only three patients. Two of these had had colonie interpositions.

\section{DISCUSSION}

In the management of ingested foreign bodies, as in other areas of clinical medicine, it ist? difficult to change established practice, especially when the patient or parents request 
investigation. This is particularly true of children who have ingested a foreign body where the parent will often not rest easy until he has had an X-ray to demonstrate that there is no problem.

The likelihood of impaction of an ingested foreign body seems to be over-estimated by both doctors and parents; this is probably because of case reports of complications in unusual cases appearing in medical journals or, indeed, in the popular press (Banks \& Potsic, 1977). Accidental swallowing of sharp foreign bodies due to the loss of touch sensation occurs with wearing dentures and is a problem now confined to adult populations (Kiff \& Hulton, 1983). This did not occur in this series. Children swallow sharp objects accidentally, even when they have normal sensations. Illingworth (1982), in her manual for the casualty officer, quotes that $10 \%$ of all objects found to be in the stomach do not subsequently pass into the duodenum. She, therefore, advocates that swallowed foreign bodies should not be treated lightly. This did not occur in this series in which only $5 \%$ of patients with primary attendance at this hospital subsequently required intervention for removal of a foreign body. In over half of the patients in whom oesophagoscopy was performed, the foreign body had already passed into the stomach and none was found. The true instance of complications following ingestion is, therefore, probably lower than the $5 \%$ quoted above, as parents are routinely advised that no treatment is required unless specific symptoms develop.

A history suggestive of aspiration must be taken seriously and correct investigation including either paired inspiration and expiration films or decubitus films is indicated (Gershel et al., 1983; Capitanio \& Kirkpatrick, 1972). In this series, three children presented with a history of a cough, gagging and respiratory distress, and, therefore, did not present a diagnostic problem. The other 12 children gave no typical history, and this is probably explained by the fact that the aspirating episode was unwitnessed or is over so quickly that the problem was not appreciated. These children presented with classical symptoms of a chest infection which failed to respond to vigorous medical treatment with antibiotics and physiotherapy. In any child in whom consolidation fails to resolve in the expected manner, an aspirated foreign body should be suspected. The authors' experience is in keeping with previous reports emphasizing the lack of early clinical signs in this group (Pyman, 1971; Blazer et al., 1980). There were four children in this series who had swallowed button batteries. The management of children who have swallowed button batteries has been the subject of many recent publications (David \& Ferguson, 1986). Numerous complications have been reported, including oesophageal burns, perforation and mercury poisoning (Shabino \& Feinberg, 1979; Langkau \& Noesges, 1985; Barros \& Barros, 1979). While serious complications can occur, they are rare and aggressive management is seldom required. A practical management protocol is fully discussed in the recent article by David \& Ferguson (1986). Radiological monitoring is employed to assess impaction in the distal oesophagus and to detect any undue delay in passage through the remaining gastrointestinal tract.

If asymptomatic children were managed expectantly on the basis of a clinical history, $55 \%$ of the referrals for $\mathrm{X}$-rays in our series could have been avoided. Non-opaque foreign bodies do not require $\mathrm{X}$-ray unless there is a history suggestive of aspiration. Routine delayed films do not alter the clinical management of the child and should be avoided. 
Information sheets available in A \& E departments advising parents to return, should specific symptoms develop, would be useful. Asymptomatic children, who later develop $\frac{\mathbb{O}}{0}$ signs of impaction, may still be detected.

Recently, there have been several publications reappraising the role of radiology in routine practices (Roberts et al., 1983; de Lacey et al., 1980a\&b; Eyes \& Evans, 1978).. $\overrightarrow{\vec{F}}$

The results of this study indicate that $\mathrm{X}$-ray examinations for ingested foreign bodieso are often misused. The introduction of the measures outlined above would approxima- $\frac{\bar{D}}{\bar{D}}$ tely halve the workload arising from the ingestion of foreign bodies and would reduce $\frac{\bar{\sigma}}{\widetilde{D}}$ unnecessary irradiation.

\section{REFERENCES}

Banks W. \& Potsic W. P. (1977) Elusive unsuspected foreign bodies in the tracheobronchial tree. Clinical! Paediatrics 16, 31-5.

Barros D'Sa E. A. \& Barros D'Sa A. A. B. (1979) Mercury battery ingestion. British Medical fournal 1, 1218. Blazer S., Naveh Y. \& Friedman A. (1980) Foreign body in the airway. American fournal of Diseases of윽 Children 134, 68-71.

Capitanio M. A. \& Kirkpatrick J. A. (1972) The lateral decubitus film: an aid to air-trapping in children. Radiology 103, 460-2.

David T. J. \& Ferguson A. P. (1986) Management of children who have swallowed button batteries. Archives of Diseases in Childhood 61, 321-2.

de Lacey G. J., Guilding A., Wignall B., Reidy J. \& Bradbrook S. (1980a) Mild head injuries: a source $8 \mathrm{f}, \sqrt{ }$ excessive radiography? Clinical Radiology 31, 457-62.

de Lacey G. J., Wignall B. K., Bradbrook S., Reidy J., Hussein S. \& Craven B. (1980b) Rationalisiżs abdominal radiography in the Accident and Emergency Department. Clinical Radiology 31, 453-5.

Eyes B. \& Evans A. F. (1978) Post-traumatic skull radiography: time for reappraisal. Lancet ii, 85-6.

Gershel K., Goldman H. S., Stein R. E. K., Shelov S. \& Ziarowski M. (1983) The usefulness of chest radiography in first asthma attacks. New England fournal of Medicine 309, 336-9.

Illingworth C. M. (1982). The Diagnosis and Primary Care of Accidents and Emergencies in Children, 2nd을 edition, pp. 33-4. Oxford, Blackwell Scientific Publications.

Kiff E. S. \& Hulton N. (1983) Small bowel perforation due to a Christmas cake decoration. British Medical fournal 27, 1923-4.

Langkau J. F. \& Noesges R.A. (1985) Esophageal burns from battery ingestion. American fournal of Emergency Medicine 3, 2265.

Pyman C. (1971) Inhaled foreign bodies in childhood: a review of 230 cases. The Medical fournal of Australia $1,62-8$.

Roberts C. J., Ennis W. P., Fowkes F. G. R. \& Mitchell M. (1983) Possible impact of audit on chest X-ray requests from surgical wards. Lancet ii, 446-7.

Shabino C. L. \& Feinberg A. N. (1979) Esophageal perforation secondary to alkaline battery ingestion. fournal of the American College of Emergency Physicians 3, 360-2.

Received 10 fuly 1986; accepted 2 November 1986 